\title{
Spatial distribution of rolled up Kelvin-Helmholtz vortices at Earth's dayside and flank magnetopause
}

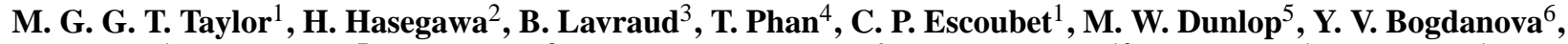 \\ A. L. Borg ${ }^{1}$, M. Volwerk ${ }^{7}$, J. Berchem ${ }^{8}$, O. D. Constantinescu ${ }^{9}$, J. P. Eastwood ${ }^{10}$, A. Masson ${ }^{1}$, H. Laakso ${ }^{1}$, \\ J. Soucek ${ }^{1,11}$, A. N. Fazakerley ${ }^{6}$, H. U. Frey ${ }^{4}$, E. V. Panov ${ }^{7}$, C. Shen ${ }^{12}$, J. K. Shi ${ }^{12}$, D. G. Sibeck ${ }^{13}$, Z. Y. Pu ${ }^{14}$, \\ J. Wang ${ }^{14}$, and J. A. Wild ${ }^{15}$ \\ ${ }^{1}$ Science and Robotic Exploration Directorate, ESA/ESTEC, Noordwijk, The Netherlands \\ ${ }^{2}$ Institute of Space and Astronautical Science, JAXA, Sagamihara, Japan \\ ${ }^{3}$ IRAP/CNRS, Toulouse, France \\ ${ }^{4}$ SPRG/SSL , Berkeley, CA, United States \\ ${ }^{5}$ RAL/STFC, Didcot, UK \\ ${ }^{6}$ MSSL/UCL, Dorking, UK \\ ${ }^{7}$ IWF, Graz, Austria \\ ${ }^{8}$ UCLA/IGPP, Los Angeles, CA, USA \\ ${ }^{9}$ ISS, Bucharest, Romania \\ ${ }^{10}$ Imperial College, London, UK \\ ${ }^{11}$ Institute of Atmospheric Physics, Bocni II 1401, 14131 Prague, Czech Republic \\ ${ }^{12}$ CSSAR, Beijing, China \\ ${ }^{13}$ NASA/GSFC, Greenbelt, MD, USA \\ ${ }^{14}$ Peking University, Beijing, China \\ ${ }^{15}$ Lancaster University, Lancaster, UK \\ Correspondence to: M. G. G. T. Taylor (mtaylor@rssd.esa.int)
}

Received: 1 March 2012 - Revised: 4 June 2012 - Accepted: 6 June 2012 - Published: 29 June 2012

\begin{abstract}
The Kelvin-Helmholtz Instability (KHI) can drive waves at the magnetopause. These waves can grow to form rolled-up vortices and facilitate transfer of plasma into the magnetosphere. To investigate the persistence and frequency of such waves at the magnetopause we have carried out a survey of all Double Star 1 magnetopause crossings, using a combination of ion and magnetic field measurements. Using criteria originally used in a Geotail study made by Hasegawa et al. (2006) (forthwith referred to as H2006), 17 candidate events were identified from the entire TC- 1 mission (covering $\sim 623$ orbits where the magnetopause was sampled), a majority of which were on the dayside of the terminator. The relationship between density and shear velocity was then investigated, to identify the predicted signature of a rolled up vortex from $\mathrm{H} 2006$ and all 17 events exhibited some level of rolled up behavior. The location of the events had a clear
\end{abstract}

dawn-dusk asymmetry, with $12(71 \%)$ on the post noon, dusk flank suggesting preferential growth in this region.

Keywords. Magnetospheric physics (Magnetopause, cusp, and boundary layers; Plasma waves and instabilities; Solar wind-magnetosphere interactions)

\section{Introduction}

The penetration of solar wind plasma across the magnetopause into the Earth's magnetosphere has been the subject of study for a number of decades. The situation for a predominantly southward directed interplanetary magnetic field (IMF) is driven by magnetic reconnection at the dayside magnetopause (Dungey, 1961). Under northward IMF, magnetic reconnection is less likely to occur at the lowlatitude magnetopause and yet evidence of ongoing transport 
is exemplified by the formation and thickening of the low latitude boundary layer (LLBL) (Eastman and Hones, 1979) and the formation of the cold dense plasma sheet (e.g. Teresawa et al., 1997). Impulsive plasma penetration has also been proposed, most recently with Cluster data (e.g. Lundin et al., 2003). Processes pertaining to plasma transport in the context of the cold dense plasma sheet are: diffusion (Treumann, 1997), poleward-of-the-cusp reconnection (Song and Russell, 1992; Øieroset et al., 2005, 2008; Lavraud et al., 2005, 2006; Li et al., 2005; Imber et al., 2006) and the Kelvin-Helmholtz instability (KHI) (Fujimoto and Teresawa, 1995; Fairfield et al., 2000; Nykiri and Otto, 2001) or a combination of each (e.g. Taylor et al., 2008). KHI activity is also observed at other planets, most recently at Saturn (Masters et al., 2010) and Mercury (Sundberg et al., 2011, 2012).

Such KHI driven waves can grow non-linearly, roll up into vortices and facilitate plasma transport into the magnetosphere, as demonstrated with simulations (Nakamura and Fujimoto, 2005; Nakamura et al., 2006) and observations by Cluster (Hasegawa et al., 2004; Nykyri et al., 2006). Takagi et al. (2006) carried out three-dimensional MHD simulations of the Kelvin-Helmholtz instability in a magnetotail flanklike geometry. They found that rolled-up vortices contained a characteristic feature when relating the velocity and density, showing a component of low-density plasma with tailward speeds exceeding that of the adjacent magnetosheath flow, and suggested that this feature could be used as a marker of the roll-up of Kelvin-Helmholtz driven vortices. H2006 presented spacecraft observations compared to 3-D MHD simulation results by Takagi et al. (2006). Four Cluster spacecraft measurements of a rolled up vortex (reported previously in Hasegawa et al., 2004) confirmed the simulation predictions of the so-called "faster-than-sheath" feature in the velocitydensity relationship. Using this feature, along with other signatures of KHI, H2006 proposed a single spacecraft method to identify rolled up vortices and carried out a survey on a large data set of 9 years of Geotail measurements. Of the 19 rolled up events identified, only 1 was found on the day side of the dawn-dusk terminator and a near symmetric distribution across both flanks, with 9 on the dawn and 10 on the dusk side of the noon-midnight meridian. This technique has been used in more recent studies (Nishino et al., 2007; Taylor et al., 2008; Hwang et al., 2011) and similar features were observed in hybrid simulations of KHI at the magnetopause (Cowee et al., 2010). In the current study we present an extension of H2006's work using the complete Double Star 1 data set.

\section{Instrumentation}

The Double Star 1 spacecraft was launched on 29 December 2003 into a $570 \times 78970 \mathrm{~km}$ equatorial orbit (Liu et al., 2005). Post commissioning observations began after February 2004, and on 14 October 2007 after completing its de- signed orbit lifetime it returned to Earth having completed around 1163 27.25-h orbits. We use data from the Hot Ion Analyzer (HIA; Rème et al., 2005), which measures full three-dimensional (3-D) ion (without mass discrimination) distribution functions in the energy range $5-32 \mathrm{keV} \mathrm{q}^{-1}$ (32 energy steps) every spin (4s), with an angular resolution of $11.25^{\circ}-22.5^{\circ}$. The moments shown here come from straight integration of the distribution functions onboard the spacecraft. The full 3-D distributions transmitted to the ground from which the distributions in this paper are drawn are accumulated over 2 spins $(8 \mathrm{~s})$ and have an angular resolution of $22.5^{\circ}-22.5^{\circ}$. We also use spin resolution magnetic field measurements from the Flux Gate Magnetometer (FGM; Carr et al., 2005). Solar wind conditions are taken from $5 \mathrm{~min}$, high resolution OMNI data (King and Papitashvili, 2005), which are provided time-shifted to the bow shock nose. We estimate an additional magnetosheath transition period (Spreiter and Stahara, 1994) of between 2-5 min based on the variation of solar wind conditions, which we translate to an overall 10 min additional lag when considering the IMF and solar wind conditions below, to avoid bow shock - magnetopause/spacecraft location convection time uncertainties.

\section{Observations}

We surveyed all TC-1 magnetopause crossings by eye, examining the behaviour of the ion moments, omni-directional energy-time spectrograms and time series of magnetic field components. We identified candidate rolled-up KHI intervals using criteria based on H2006. These were (1) clear quasi-periodic fluctuations with period $1-5 \mathrm{~min}$ in the spectrograms, bulk plasma parameters and magnetic field; (2) to restrict events to having at least 5 wave periods; (3) IMF orientation is continuously northward (with a clock angle $\left(\theta_{\mathrm{IMF}}=\arctan \left(B_{\mathrm{y}} / B_{\mathrm{Z}}\right) \mathrm{GSM}\right)$ between $\pm 70^{\circ}$ for the entire the time period, based on the OMNI IMF data propagated to the magnetopause as described above); (4) a sufficient number of low density measurements had associated anti-sunward speed that was higher than that of the magnetosheath. We note that H2006 do not quantify this level specifically, which we have attempted to do below. Criterion (3) is imposed to avoid high magnetic shear at the magnetopause boundary and hence mis-interpreting low density, high speed flows generated through reconnection instead of rolled up vortices. To impose criterion (4), a shear flow direction for the crossings was defined from taking the average of all flow directions during a single event. This shear direction was also compared to the local magnetosheath flow in each case and found to be in agreement (directionally) to within $10^{\circ}$, suggesting that the shear direction was reasonably tangential to the nominal magnetopause surface.

Figure 1 shows an example magnetopause crossing on 14 April 2007. Panel (a) shows an omnidirectional ion differential energy flux spectrogram, panel (b) the magnetic 


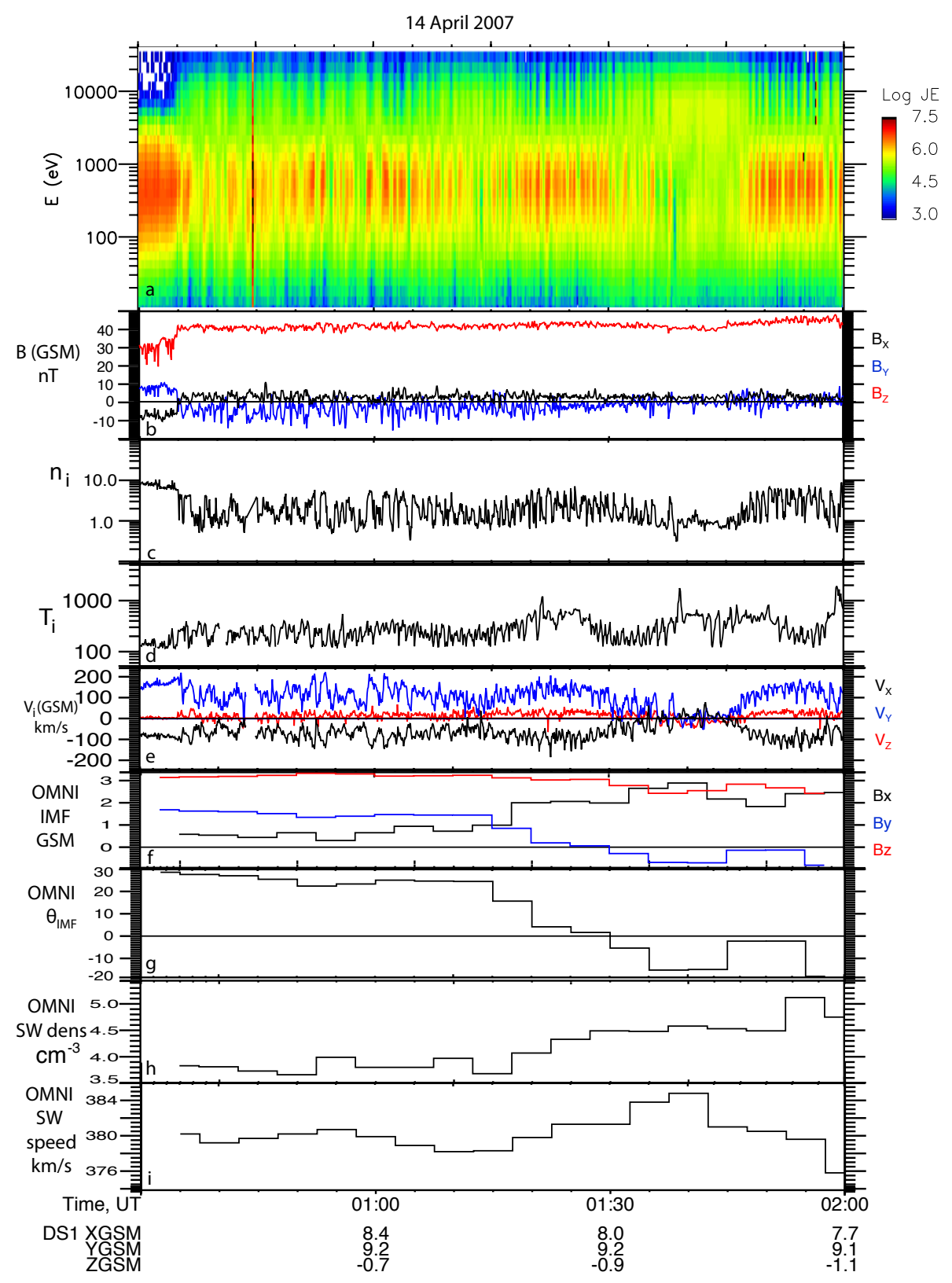

Fig. 1. An example magnetopause crossing on 14 April 2007. Panel (a) shows an omnidirectional ion differential energy flux spectrogram, panel (b) the magnetic field GSM components, panels (c)-(e) show the ion density, temperature (parallel and perpendicular components) and GSM velocity components. Panels (f)-(i), show OMNI data, upstream solar wind conditions (where $\left.\theta_{\mathrm{IMF}}=\arctan \left(B_{\mathrm{y}} / B_{\mathrm{Z}}\right) \mathrm{GSM}\right) \operatorname{time}$ shifted to the magnetopause, by taking the OMNI parameters shifted to the Bow Shock (King and Papitashvili, 2005) and adding an extra $10 \mathrm{~min}$ as described in the text.

field GSM components, panels (c)-(e) show the ion density, scalar temperature and GSM velocity components. The final four panels, (f)-(i), show OMNI data, upstream solar wind conditions (where $\theta_{\mathrm{IMF}}=\arctan \left(B_{\mathrm{y}} / B_{\mathrm{z}}\right) \mathrm{GSM}$ ) time shifted to the magnetopause, by taking the OMNI parameters shifted to the Bow Shock (King and Papitashvili, 2005) and adding an extra $10 \mathrm{~min}$ as described above. The figure shows the passage of Double Star 1 from the magnetosheath into a magnetopause boundary layer and adjacent hotter tenuous magnetospheric like population, with persistent quasi-periodic transitions between the different populations. At around 00:35 UT the spacecraft crossed the magnetopause into a dynamic magnetospheric boundary layer, with persistent wave-like signatures characterized by transitions from cool, denser magnetosheath-like boundary layer plasma to hot tenuous population of the magnetosphere. The plasma conditions are far from laminar with clear wave like signatures in the magnetic field and the ion data. During the 


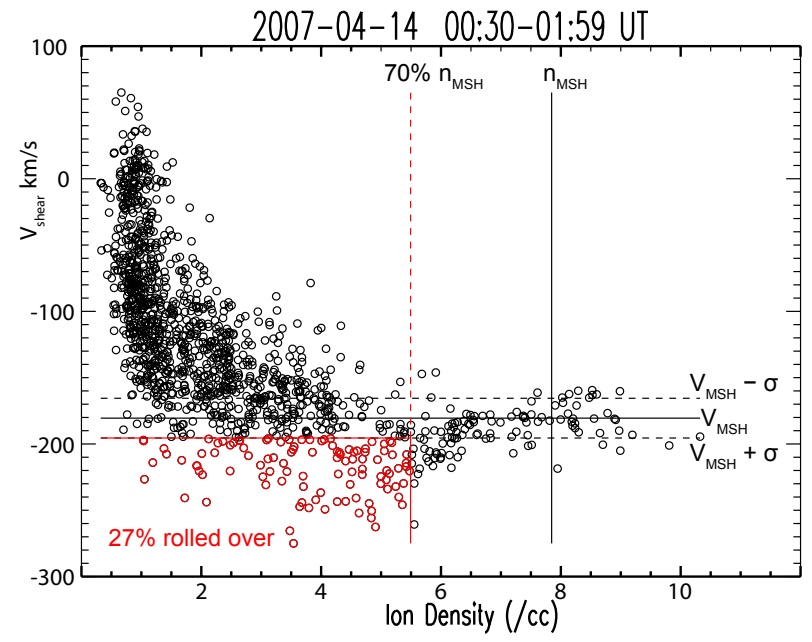

Fig. 2. A plot of shear velocity versus density for the event in Fig. 1. The plot is described in the text.

entire period the interplanetary magnetic field is predominantly northward. The crossing shown in Fig. 1 is rather typical of the events examined, which we list in full in Table 1.

Table 1 indicates all events adhering to criteria 1-3 and provides a number of characteristics for each event, in particular the level to which the event adheres to criterion 4 by the column $\%_{\mathrm{RO}}$, which is discussed below. The times in Table 1 indicate the period over which the analysis for criterion 4 was carried out. Of all crossings, only events $5+6$ are closely spaced in time and location, as a result of a reexit into the magnetosheath during a particular orbit. We note that event 3 has been discussed at some length in Taylor et al. (2008) and Taylor and Lavraud (2008). In addition to $\%_{\mathrm{RO}}$, for each event the $\beta_{\text {magnetosheath }}$, solar wind Alfvén Mach number $M_{\mathrm{A}}(\mathrm{OMNI})$, speed and density along with the TC1 GSM location are listed. We note that $\beta_{\text {magnetosheath }}$ is the value nearest to the event in question and may in some cases be very close to the boundary itself so not always "pristine" magnetosheath. The "Extended NW IMF" column indicates whether the IMF was northward for a least $1 \mathrm{~h}$ before the period examined (where the $*$ indicates that $\theta_{\mathrm{IMF}}$ was within $20^{\circ}$ of pure northward for the entire preceding hour) and is followed by the average wave period and associated wavelength (based on the magnetosheath velocity). The next column includes a ratio of the distance from the subsolar point to the wavelength, where $d_{\text {subsolar }}$ is an approximate distance of the event from the sub-solar point. The mixing status indicates the ion mixing status in a similar manner to H2006. "Mixed" indicates a significant amount of cool magnetosheath-like plasma (density $>1 / \mathrm{cc}$ ) was observed on the magnetospheric side of the magnetopause. "Weakly" indicates that magnetosheath like plasma was observed on the magnetospheric side, but with densities lower than $1 / \mathrm{cc}$. $\Theta_{\mathrm{AZI}}=\tan ^{-1}\left(\mathrm{IMF} B_{\mathrm{y}} / \mathrm{IMF} B_{\mathrm{x}}\right)$ indicates the orientation of the IMF in the ecliptic plane, where multiple entries indicate
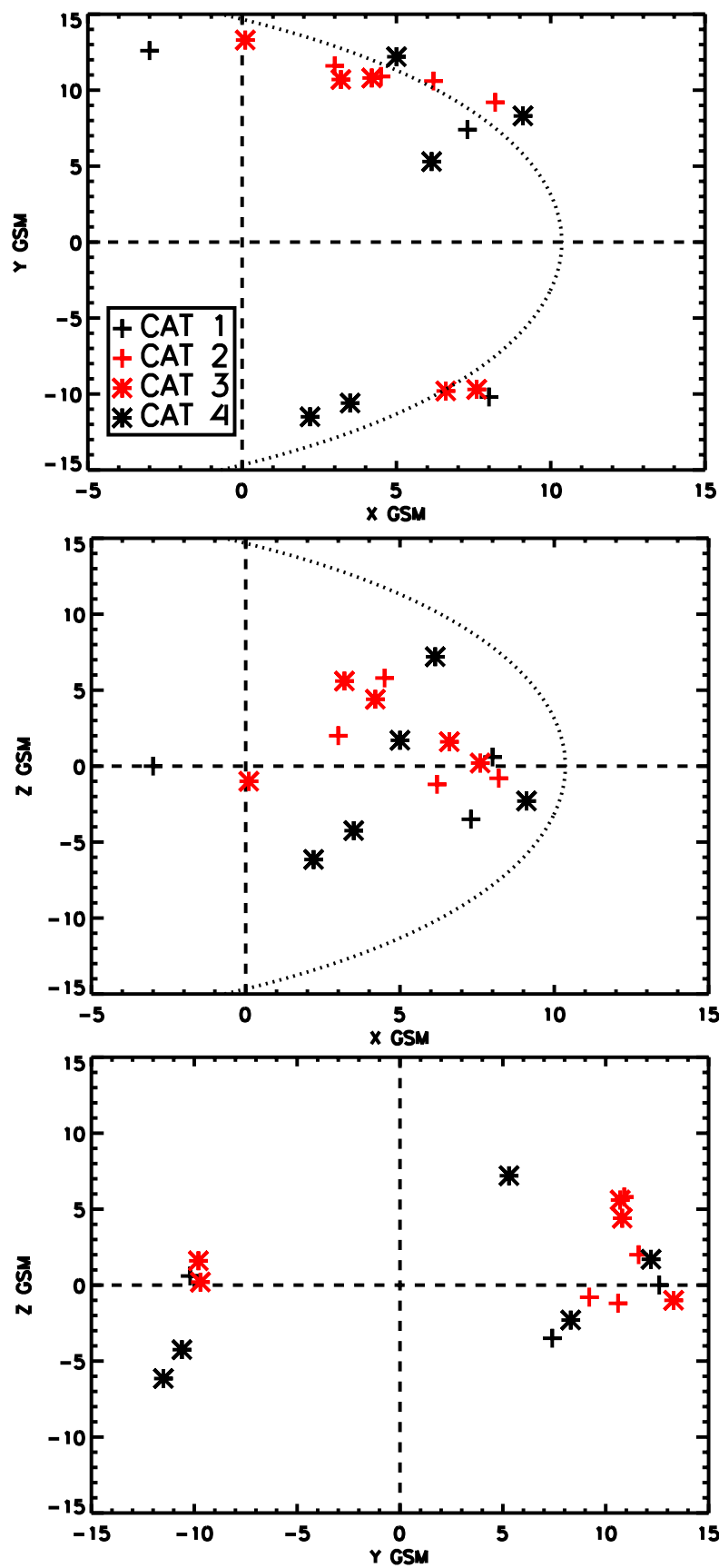

Fig. 3. The location of all the events listed in Table 1 in Geocentric Solar Magnetospheric (GSM) coordinates, subdivided into varying levels of $\%_{\mathrm{RO}}$ : (Category 1$) \%_{\mathrm{RO}}>40$, (Category 2 ) $30<\%_{\mathrm{RO}}<$ 40, (Category 3 ) $10<\%_{\mathrm{RO}}<20 \%$ and (Category 4 ) $\%_{\mathrm{RO}}<10 \%$.

variations in the IMF. $\Theta_{\mathrm{AZI}}$ is used in the final column to determine the nature of the shock upstream of each event, either parallel or perpendicular (or a mixture of both due to field rotations). The yellow highlight indicates the pre-noon/dawn events. 


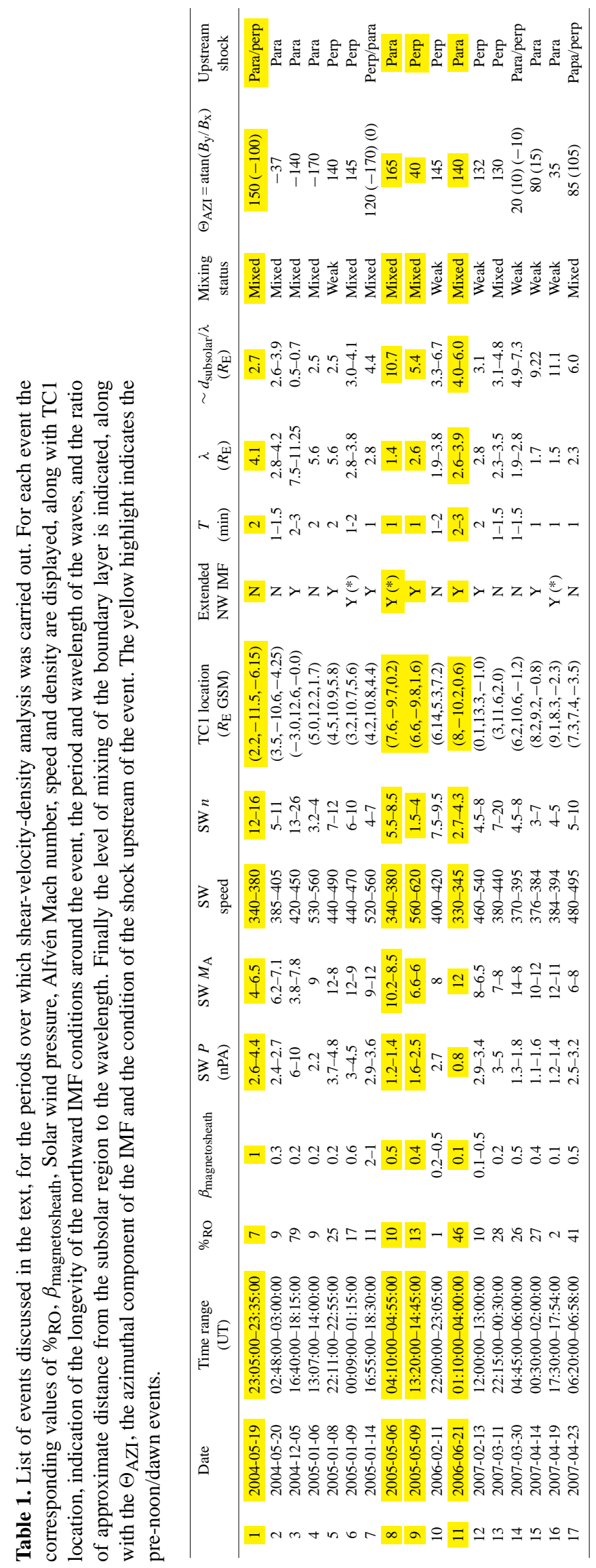


To attempt to quantify criterion 4 and the rolled up nature of the crossings, we introduce a parameter, $\%_{\mathrm{RO}}$, to quantify the "faster-than-sheath" characteristic. To do this, as in H2006, the shear velocity and density for each period were plotted to find "faster-than-sheath" features. Figure 2 shows such a plot for the same data as were introduced in Fig. 1. As mentioned previously, $V_{\text {shear }}$ was defined by taking the average flow velocity for the entire period, as implemented in H2006. To obtain the faster-than-sheath component, we define a magnetosheath density, $n_{\mathrm{MSH}}$, as $80 \%$ of the maximum density from the entire period (the vertical solid line in Fig. 2). The characteristic sheath velocity, $V_{\text {MSH }}$ (horizontal black line in Fig. 2) is derived from the average shear velocity (with standard deviation, $\sigma$ ) for all points above $n_{\mathrm{MSH}}$. From the faster-than-sheath features discussed in H2006, values between $60 \%$ and $75 \%$ of the characteristic magnetosheath density were considered. We have used a value of $70 \% n_{\mathrm{MSH}}$ as the upper threshold for density in the current study (indicated by the vertical red line in Fig. 2). The faster-than-sheath component of the data is then defined as all points with $n<70 \% n_{\mathrm{MSH}}$ and with a velocity threshold $V_{\text {shear }}<\left(V_{\mathrm{MSH}}-\sigma\right)$. This region is indicated in Fig. 2 by the region bounded by solid red lines. To provide a generic identification and quantification of such a "rolled-over" characteristic, we relate these low density-high speed components to all points with magnetosheath-like velocities $\left(V_{\text {shear }}<V_{\mathrm{MSH}}+\sigma\right.$ (indicated by the uppermost horizontal dashed black line in Fig. 2)) to obtain a percentage value, $\%_{\text {RO }}$. The outcome of the examination of criterion 4 is shown for all events in Table 1 and shows that all events have evidence of some level of rolling up. Relaxing the velocity threshold from $\left(V_{\mathrm{MSH}}-\sigma\right)$ to $V_{\mathrm{MSH}}$ made no apparent change to the results, other than increasing the value of $\%_{\mathrm{RO}}$ for each event.

\section{Discussion and conclusions}

There is a clear asymmetry in the spatial distribution of the events, with 12 of the 17 (71\%) events on the post noondusk hemisphere and only 5 on the dawn, pre-noon side. Figure 3 shows the location of all the events listed in Table 1 in Geocentric Solar Magnetospheric (GSM) coordinates and sub-divides them in terms of the value of $\%_{\mathrm{RO}}$ as follows: (Category 1) $\%_{\text {RO }}>40$, (Category 2) $30<\%$ RO $<40$, (Category 3 ) $10<\%_{\text {RO }}<20 \%$ and (Category 4$) \%_{\text {RO }}<10 \%$. All events, apart from the 5 December 2004, lay sunward of the terminator, suggesting rather rapid evolution of the KHI for these events, evolving to a non-linear state in only a few wavelengths. There is no clear ordering in $x$ GSM and categories of $\%_{\mathrm{RO}}$ on the dusk flank. On the dawn flank, somewhat counter-intuitively, $\%_{\text {RO }}$ decreases with increasing distance from the sub solar region. Indeed, for events 5 and 6 , which are closely located in time, $\%$ RO also reduces with distance from the sub-solar point. As discussed by H2006, the appearance of the v-n plot is dependent on the trajectory of the spacecraft through the vortex, and therefore may not necessarily contain all the points from the simulated plots in Tagaki et al. (2006). H2006 suggested that the "faster-thansheath" high velocity-low density data points in v-n plots are most likely obtained from the magnetosheath side of the vortex centre and that clustering of data points at low density and lower velocities could indicate sampling of the inner region of the vortex centre (Hwang et al., 2011). Hybrid simulations by Cowee et al. (2010) have indicated that "faster-thansheath" signatures may be suppressed with higher magnetic shear as the low-density flows are not accelerated as much. In addition, they found no "faster-than-sheath" signature for cases with low-density gradients across the boundary. So we consider that the spatial variation of $\%_{\mathrm{RO}}$ of each event is not simply related to the distance from the sub-solar point, but also to specific conditions of the boundary at each event and to the sampling of the rolled-up vortex structure itself.

As shown in Table 1, events have a low $\beta_{\text {magnetosheath and }}$ solar wind $M_{\mathrm{A}}$ values are all $>4$. There is no clear correlation for either $M_{\mathrm{A}}$ or $\beta_{\text {magnetosheath with } \%_{\mathrm{RO}} \text {. Lavraud }}$ and Borovsky (2008) discussed the possible implications of low solar wind $M_{\mathrm{A}}$ and $\beta_{\text {magnetosheath, where increased flow }}$ shears may be expected at the flank magnetopause. Low $\beta_{\text {magnetosheath }}$ and hence similar magnetic and plasma conditions on either side of the magnetopause boundary, would result in KHI growing for any velocity shear value (with growth rate being proportional to this shear magnitude). However, they also noted that low values of $\beta_{\text {magnetosheath }}$, as observed in this study would result in stronger magnetic tension forces to act against the growth of the KHI. The lack of correlation between KHI vortex properties (location and $\%_{\mathrm{RO}}$ ) and upstream conditions suggest a counteracting interplay between larger magnetopause velocity shear and the stabilization effect of magnetic tension forces affecting KHI wave growth.

All events in this study display some level of mixing at the boundary (Table 1). If we consider this to be the signature of a pre-existing boundary layer, this may have aided the excitation of the KHI (Hasegawa et al., 2009), presumably formed by double-lobe reconnection (Song and Russell, 1992). Unlike $\mathrm{H} 2006$, who related weaker mixing to periods of weaker or shorter-lived northward IMF, we find weaker mixing for events with extended $(>1 \mathrm{~h})$ northward IMF and in the case of event 17 after periods of strongly northward IMF $\left(\theta_{\mathrm{IMF}}\right.$ within $20^{\circ} \mathrm{NW}$ ). We note that no weakly mixed events occur in the pre-noon sector and that there is a weak tendency for the more weakly mixed events to occur close to the sub-solar region (larger $X_{\mathrm{GSM}}$ ), perhaps indicating broadening of the boundary layer (Mitchell et al., 1987; Foullon et al., 2008). In terms of wave period and wavelength, there is weak tendency for period and wavelength to increase with distance from the subsolar point, in a similar manner to that reported in H2006. This is more clearly exemplified in Table 1, which shows the ratio of the distance from the subsolar point to the wavelength of the event. The weaker mixed events also tend 
to have a smaller period and wavelength, perhaps reflecting the relationship between wavelength and growth rate and the thickness of the boundary layer (e.g. Miura and Pritchett, 1982).

A number of studies have reported a dawn-dusk asymmetry in the characteristics of the magnetosphere, for example in the plasma sheet and LLBL under northward IMF (Fujimoto et al., 1998, 2002; Wing and Newell, 2002; Hasegawa et al., 2003). These studies reported the existence of a twocomponent ion energy distribution, comprised of a cold magnetosheath and a hotter magnetospheric population on the dusk side and a more condensed/mixed distribution on the dawn flank. Nishino et al. (2011) reported simultaneous observations of KHI driven waves at both flanks of the magnetosphere, with simultaneous conjugate flank observations from Geotail and Cluster. The macroscopic appearance of the vortex structures themselves was roughly symmetric, although at the microscopic level there were clear differences, with a mixed like appearance of distributions around the dawn vortices and a two-component distribution found on the dusk side. Dawn-dusk asymmetries have also been reported in ground based PC5 wave activity (Baker et al., 2003).

Such asymmetry may be seeded by perturbations downstream of the quasi-parallel bow shock (Miura, 1992) for the dawn flank and finite Larmor radius effects on the dusk (Huba, 1996) or dawn (Nagano, 1979) flank. In the magnetosheath, Němeček et al. (2003), Paularena et al. (2001), Longmore et al. (2005) have shown higher ion fluxes and velocities and lower densities on the dusk flank compared to the dawn, with no link to IMF orientation. However, Paularena et al. (2001) showed a solar cycle variation, with no correlation during solar maximum but some correlation with solar wind conditions during solar minimum. In the current study we examined the IMF azimuthal clock angle $\left(\Theta_{\mathrm{AZI}}=\tan ^{-1}\right.$ (IMF $\left.B_{\mathrm{y}} / \mathrm{IMF} B_{\mathrm{x}}\right)$ ) to infer a likely orientation of the IMF to the nominal bow shock normal upstream of each event. We found no correlation with the observed asymmetry, with a rather balanced distribution of either quasi-parallel or quasiperpendicular or mixed conditions, as indicated in Table 1.

MHD KHI simulations by Collado-Vega et al. (2007) show a majority of KHI driven vortices on the dawnside for a particular case study. H2006 reported a nearly symmetric distribution of rolled up vortices over the dawn and dusk flank, with 9 events post-noon/dusk and 10 events pre-noon/dawn, with all except one anti-sunward of the terminator. We plot these events along side those of the current study in Fig. 4, with Geotail locations in red and TC1 locations in black. Combining both datasets, of the total 36 events, 21 (58\%) are found on the dusk side. As presented above, the TC1 mission was in operation for just over 3.5 years. Initial operations began in late February 2004, coinciding with apogee being in the morning sector and representing the first morning flank season in the current study, as the spacecraft orbit precessed clockwise, towards earlier local time. If we divide the coverage into dawn/morning and afternoon/evening and
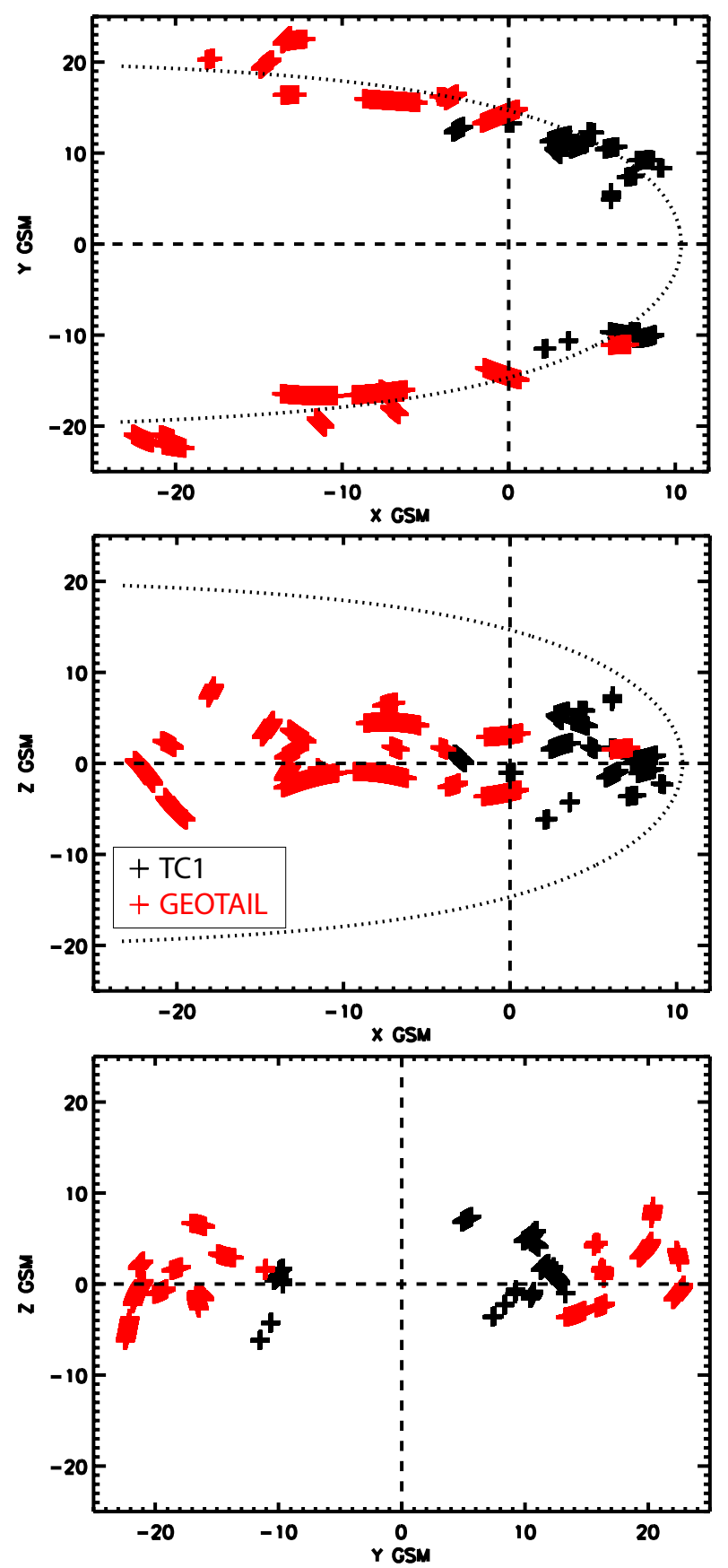

Fig. 4. Location of all events from current study combined with Geotail events from H2006m with Geotail locations in red and TC1 locations in black.

we consider the entire period surveyed in this paper, from 24 February 2004-4 May 2007, we find that the spacecraft sampled both regions on three occasions each (we note that although the mission continued up to October 2007 before re-entering, data coverage was rather sparse during the period after 4 May). The Geotail data used in H2006 were taken over a period of 9 years from 1995-2003 and also provided 
an equal sampling of both flanks, so we do not believe the dawn-dusk asymmetry is due to a sampling issue.

From Fig. 4, a clearer asymmetry can be seen with respect to the terminator, with the majority of TC1 events on the dayside and on the nightside for Geotail. The smaller apogee of TC1 $\left(\sim 12 R_{\mathrm{E}}\right)$ prevented sampling of the flanks at larger distances anti-sunward of the terminator. For Geotail, H2006 examined the period 1995-2003, during the mission's "near-tail" phase in which the apogee of the spacecraft orbit was $30 R_{\mathrm{E}}$, providing coverage for both dayside and nightside magnetopause crossings. H2006 only used 12-s resolution data from the LEP-EA instrument (Mukai et al., 1994) as this instrument gives more reliable data in the boundary layer than LEP-SW. Bulk parameter calculations are automatically selected from either LEP-EA or LEP-SW according to the intensity of ion fluxes and for the magnetosheath and outer boundary, this would likely be from LEP-SW. As the faster-than-sheath data points are expected to be on the magnetosheath side of the boundary, it is likely that a number of candidate events were not considered thereby explaining the lack of events on the dayside reported in H2006. It is not likely that the incident angle of the orbit with the magnetopause and hence time period in the vicinity of the boundary could play a role. The smaller apogee of the TC1 orbit provides more tangential or skimming crossings on the dayside for that phase of the orbit (near apogee) compared with the more perpendicular incidence for Geotail. But the higher perigee $\left(\sim 10 R_{\mathrm{E}}\right)$ of the Geotail orbit would result in similar types of incidence nearer perigee, so it is more likely an issue with instrument mode than orbit coverage. Figure 4 and hence the combination of TC1 events from this study and Geotail events from H2006 provides a complete picture of the occurrence of KHI vortices under northward IMF conditions, suggesting that a dawn-dusk asymmetry in rolled up KHI driven vortices exists on the dayside but disappears antisunward of the terminator.

The various conditions discussed above could affect the growth rate, stability and initiation of the KHI at the magnetopause. In addition, magnetospheric-ionospheric coupling must be considered when examining the evolution of KHI waves (Miura and Kan, 1992). Wei and Lee (1993) investigated KHI driven vortices at the LLBL and their associated field aligned currents linking to the ionosphere. They found that competing effects of the formation and driving of the vortices at the LLBL with the dissipation at the ionosphere led to limited regions of strong vortex formation, namely in the post noon sector and associated them with bright auroral spots in the same local time sector. This, along with the vorticity enhancements on growth rates from finite Larmor radius effects could result in the dayside asymmetry observed in the current study. We note that Sundberg et al. (2012) have recently reported a similar asymmetry in the occurrence of KHI driven waves at the magnetopause of Mercury, where all events were found on the post noon dusk side magnetopause. Why such an asymmetry in the current study does not con- tinue to the tail, could then be due to the formation of secondary KHI and Rayleigh Taylor instabilities (Cowee et al., 2009), which would work to break down the vortices formed at the dayside. These effects are dependent on the magnetic shear and density profile across the magnetopause and may have different magnitudes on each flank, ultimately bringing a more uniform scenario at both the magnetopause flanks, anti-sunward of the terminator.

\section{Summary}

We have surveyed the entire Double Star 1 mission to find magnetopause crossings exhibiting behavior akin to the rolling up of Kelvin-Helmholtz unstable waves. This survey was implemented using a technique proposed in Takagi et al. (2006) and implemented in spacecraft data by Hasegawa et al. (2006). We have found an asymmetric distribution of KHI driven rolled up vortices, with $70 \%$ (12 out of 17) of events occurring at the post noon magnetopause. All but one of these events was found on the dayside magnetosphere, suggesting rapid evolution of the KHI to a non-linear state. We have attempted to quantify the magnitude of the rolling up in terms of a $\%$ of low-density higher velocity data points or $\%_{\mathrm{RO}}$ but found no obvious tendency with location. We found no clear differences either in terms of wavelength, $\beta_{\text {magnetosheath }}$, or a number of solar wind parameters including $M_{\mathrm{A}}$ or inferred bow shock characteristics upstream of the events.

To provide a broader picture of vortices we combined the current study results with those from H2006, resulting in the asymmetry to reduce to $62 \%$ ( 21 out of 34 ). This also showed the asymmetry to be clearly limited to the dayside, with the post-noon sector providing the best location for rolled up vortices. A number of factors could be invoked to provide preferential KHI onset on either flank, in the case of the dusk flank, an ortho-Parker spiral configuration would provide quasi-parallel conditions at the bow shock to seed KHI growth, along with long-term average trends of higher ion fluxes and velocities in the magnetosheath. The dayside weighting of the asymmetry pertains to a driver sunward of the terminator, with possible link to the dayside coupling of the magnetosphere/ionosphere in the post noon sector. Why such an asymmetry does not continue into the tail could then be a combination of various local (magnetopause) and connectivity (ionosphere) conditions, along with the varying contributions from secondary processes, again related to the local plasma conditions. Linking the asymmetry reported here with those observed within the magnetosphere itself should be the target of a future study incorporating simultaneous observations of the magnetosphere and magnetopause. 
Acknowledgements. The authors would like to thank the Double Star instruments teams, along with OMNIweb, for provision of data and AMDA for its data visualisation and analysis tool. Part of this study was carried out under the auspices of the International Space Science Institute in Bern, Switzerland and the "Conjugate response of the dayside magnetopause and dawn/dusk flanks using ClusterTHEMIS conjunction and Ground based observation" international team. MGGTT would like to thank A. Masters and M. Nishino for useful discussions.

Topical Editor I. A. Daglis thanks J. De Keyser and I. J. Rae for their help in evaluating this paper.

\section{References}

Baker, G., Donovan, E., and Jackel, B.: A comprehensive survey of auroral latitude Pc5 pulsation characteristics, J. Geophys. Res., 108, 1384, doi:10.1029/2002JA009801, 2003.

Carr, C., Brown, P., Zhang, T. L., Gloag, J., Horbury, T., Lucek, E., Magnes, W., O’Brien, H., Oddy, T., Auster, U., Austin, P., Aydogar, O., Balogh, A., Baumjohann, W., Beek, T., Eichelberger, H., Fornacon, K.-H., Georgescu, E., Glassmeier, K.-H., Ludlam, M., Nakamura, R., and Richter, I.: The Double Star magnetic field investigation: instrument design, performance and highlights of the first year's observations, Ann. Geophys., 23, 2713-2732, doi:10.5194/angeo-23-2713-2005, 2005.

Collado-Vega, Y. M., Kessel, R. L., Shao, X., and Boller, R. A.: MHD flow visualization of magnetopause boundary region vortices observed during high-speed streams, J. Geophys. Res., 112, A06213, doi:10.1029/2006JA012104, 2007.

Cowee, M. M., Winske, D., and Gary, S. P.: Two-dimensional hybrid simulations of superdiffusion at the magnetopause driven by Kelvin-Helmholtz instability, J. Geophys. Res., 114, A10209, doi:10.1029/2009JA014222, 2009.

Cowee, M. M., Winske, D., and Gary, S. P.: Hybrid simulations of plasma transport by Kelvin-Helmholtz instability at the magnetopause: Density variations and magnetic shear, J. Geophys. Res., 115, A06214, doi:10.1029/2009JA015011, 2010.

Dungey, J. W.: Interplanetary magnetic field and auroral zones, Phys. Rev. Lett., 6, 47-48, 1961.

Eastman, T. E. and Hones Jr., E. W.: Characteristics of the magnetospheric boundary layer and magnetopause layer as observed by IMP 6, J. Geophys. Res., 84, 2019-2028, 1979.

Fairfield, D. H., Otto, A., Mukai, T., Kokubun, S., Lepping, R. P., Steinberg, J. T., Lazarus, A. J., and Yamamoto, T.: Geotail observations of the Kelvin-Helmholtz instability at the equatorial magnetotail boundary for parallel northward fields, J. Geophys. Res., 105, 21159-21173, 2000.

Foullon, C., Farrugia, C. J., Fazakerley, A. N., Owen, C. J., Gratton, F. T., and Torbert, R. B.: Evolution of Kelvin-Helmholtz activity on the dusk flank magnetopause, J. Geophys. Res., 113, A11203, doi:10.1029/2008JA013175, 2008.

Fujimoto, M. and Teresawa, T.: Anomalous ion mixing within an MHD scale Kelvin-Helmholtz vortex, 2. Effects of inhomogeneity, J. Geophys. Res., 100, 12025-12033, doi:10.1029/94JA02219, 1995.

Fujimoto, M., Terasawa, T., Mukai, T., Saito, Y., Yamamoto, T., and Kokubun, S.: Plasma entry from the flanks of the near-Earth magnetotail: Geotail observations, J. Geophys. Res., 103, 43914408, doi:10.1029/97JA03340, 1998.
Fujimoto, M., Mukai, T., and Kokubun, S.: Cold-dense plasma sheet and hot dense ions in the inner-magnetosphere, Adv. Space Res., 30, 2279-2288, 2002.

Hasegawa, H., Fujimoto, H., Maezawa, K., Saito, Y., and Mukai, T.: Geotail observation of the dayside outer boundary region: Interplanetary magnetic field control and dawn-dusk asymmetry, J. Geophys. Res., 108, 1163, doi:10.1029/2002JA009667, 2003.

Hasegawa, H., Fujimoto,M., Phan, T.-D., Rème, H., Balogh, A., Dunlop, M. W., Hashimoto, C., and TanDokoro, R.: Transport of solar wind into Earth's magnetosphere through rolled-up KelvinHelmholtz vortices, Nature, 430, 755-758, 2004.

Hasegawa, H., Fujimoto, M., Takagi, K., Saito, Y., Mukai, T., and Rème, H.: Single Spacecraft detection of rolled-up KelvinHelmholtz vortices at the flank magnetopause, J. Geophys. Res., 111, A09203, doi:10.1029/2006JA011728, 2006.

Hasegawa, H., Retinò, A., Vaivads, A., Khotyaintsev, Y., André, M., Nakamura, T. K. M., Teh, W.-L., Sonnerup, B. U. Ö., Schwartz, S. J., Seki, Y., Fujimoto, M., Saito, Y., Rème, H., and Canu, P.: Kelvin-Helmholtaz waves at the Earth's magnetopause: Multiscale development and associated reconnection, J. Geophys. Res., 114, A12207, doi:10.1029/2009JA014042, 2009.

Huba, J. D.: The Kelvin-Helmholtz instability Finite Larmor radius magnetohydrodynamics, Geophys. Res. Lett., 23, 2907-2901, 1996.

Hwang, K.-J., Kuznetsova, M. M., Sahraoui, F., Goldstein, M. L., Lee, E., and Parks, G. K.: Kelvin-Helmholtz waves under southward interplanetary magnetic field, J. Geophys. Res., 116, A08210, doi:10.1029/2011JA016596, 2011.

Imber, S. M., Milan, S. E., and Hubert, B.: The auroral and ionospheric flow signatures of dual lobe reconnection, Ann. Geophys., 24, 3115-3129, doi:10.5194/angeo-24-3115-2006, 2006.

King, J. H. and Papitashvili, N. E.: Solar wind spatial scales in and comparisons of hourly Wind and ACE plasma and magnetic field data, J. Geophys. Res., 110, A02104, doi:10.1029/2004JA010649, 2005.

Lavraud, B. and Borovsky, J. E.: Altered solar wind-magnetosphere interaction at low Mach numbers: Coronal mass ejections, J. Geophys. Res., 113, A00B08, doi:10.1029/2008JA013192, 2008.

Lavraud, B., Thomsen, M. F., Taylor, M. G. G. T., Wang, Y. L., Phan, T. D., Schwartz, S. J., Elphic, R. C., Fazakerley, A., Rème, H., and Balogh, A.: Characteristics of the magnetosheath electron boundary layer under northward interplanetary magnetic field: Implications for high-latitude reconnection, J. Geophys. Res., 110, A06209, doi:10.1029/2004JA010808, 2005.

Lavraud, B., Thomsen, M. F., Lefebvre, B., Schwartz, S. J., Seki, K., Phan, T. D., Wang, Y. L., Fazakerley, A., Rème, H., and Balogh, A.: Evidence for newly closed magnetosheath field lines at the dayside magnetopause under northward IMF, J. Geophys. Res., 111, A05211, doi:10.1029/2005JA011266, 2006.

Li, W., Raeder, J., Dorelli, J., Øieroset, M., and Phan, T. D.: Plasma sheet formation during long period of northward IMF, Geophys. Res. Lett., 32, L12S08, doi:10.1029/2004GL021524, 2005.

Liu, Z. X., Escoubet, C. P, Pu, Z., Laakso, H., Shi, J. K., Shen, C., and Hapgood, M.: The Double Star mission, Ann. Geophys., 23, 2707-2712, doi:10.5194/angeo-23-2707-2005, 2005.

Longmore, M., Schwartz, S. J., Geach, J., Cooling, B. M. A., Dandouras, I., Lucek, E. A., and Fazakerley, A. N.: Dawn-dusk asymmetries and sub-Alfvénic flow in the high and low latitude mag- 
netosheath, Ann. Geophys., 23, 3351-3364, doi:10.5194/angeo23-3351-2005, 2005.

Lundin, R., Sauvaud, J.-A., Rème, H., Balogh, A., Dandouras, I., Bosqued, J. M., Carlson, C., Parks, G. K., Möbius, E., Kistler, L. M., Klecker, B., Amata, E., Formisano, V., Dunlop, M., Eliasson, L., Korth, A., Lavraud, B., and McCarthy, M.: Evidence for impulsive solar wind plasma penetration through the dayside magnetopause, Ann. Geophys., 21, 457-472, doi:10.5194/angeo-21457-2003, 2003.

Masters, A., Achilleos, N., Kivelson, M. G., Sergis, N., Dougherty, M. K., Thomsen, M. F., Arridge, C. S., Krimigis, S. M., McAndrews, H. J., Kanani, S. J., Krupp, N., and Coates, A. J.: Cassini observations of a Kelvin-Helmholtz vortex in Saturn's outer magnetosphere, J. Geophys. Res., 115, A07225, doi:10.1029/2010JA015351, 2010.

Miura, A.: Kelvin-Helmholtz Instability at the Magnetospheric Boundary: Dependence on the Magnetosheath Sonic Mach Number, J. Geophys. Res., 97, 10655-10675, 1992.

Miura, A. and Kan, J. R.: Line-tying effects on the KelvinHelmholtz instability, Geophys. Res. Lett., 19, 1611-1614, 1992.

Miura, A. and Pritchett, P. L.: Nonlocal stability analysis of the MHD Kelvin-Helmholtz instability in a compressible plasma, J. Geophys. Res., 87, 7431-7444, 1982.

Mitchell, D. G., Kutchko, F., Williams, D. J., Eastman, T. E., and Frank, L. A.: An extended study of the low-latitude boundary layer on the dawn and dusk flanks of the magnetosphere, J. Geophys. Res., 92, 7394-7404, 1987.

Mukai, T., Machida, S., Saito, Y., Hirahara, M., Terasawa, T., Kaya, N., Obara, T., Ejiri, M., and Nishida, A.: The Low Energy Particle (LEP) experiment onboard the GEOTAIL satellite, J. Geomag. Geoelectr., 46, 669-692, 1994.

Nagano, H.: Effect of finite ion Larmor radius on the KelvinHelmholtz instability of the magnetopause, Planet. Space Sci., 27, 881-884, 1979.

Nakamura, T. K. M., and Fujimoto, M.: Magnetic reconnection within rolled-up MHD-scale Kelvin-Helmholtz vortices: Twofluid simulation including finite electron inertial effects, Geophys. Res. Lett., 32, L21102, doi:10.1029/2005GL023362, 2005.

Nakamura, T. K. M., Fujimoto, M., and Otto, A.: Magnetic reconnection induced by weak Kelvin-Helmholtz instability and the formation of the low-latitude boundary layer, Geophys. Res. Lett., 33, L14106, doi:10.1029/2006GL026318, 2006.

Němeček, Z., Hayosh, M., Šafránková, J., Zastenker, G. N., and Richardson, J. D.: The Dawn-dusk asymmetry of the magnetosheath: Interball-1 observations, Adv. Space Res., 31, 13331340, 2003

Nishino, M. N., Fujimoto, M., Ueno, G., Mukai, T., and Saito, Y.: Origin of temperature anisotropies in the cold plasma sheet: Geotail observations around the Kelvin-Helmholtz vortices, Ann. Geophys., 25, 2069-2086, doi:10.5194/angeo-252069-2007, 2007.

Nishino, M. N., Hasegawa, H., Fujimoto, M., Saito, Y., Mukai, T., Dandouras, I., Rème, H., Retinò, A., Nakamura, R., Lucek, E., and Schwartz, S.: A case study of Kelvin-Helmholtz vortices on both flanks of the Earth's magnetotail, Planet. Space Sci., 59, 502-509, doi:10.1016/j.pss.2010.03.011, 2011.

Nykyri, K. and Otto, A.: Plasma transport at the magnetospheric boundary due to reconnection in KelvinHelmholtz vortices, Geophys. Res. Lett., 28, 3565-3568,
doi:10.1029/2001GL013239, 2001.

Nykyri, K., Otto, A., Lavraud, B., Mouikis, C., Kistler, L. M., Balogh, A., and Rème, H.: Cluster observations of reconnection due to the Kelvin-Helmholtz instability at the dawnside magnetospheric flank, Ann. Geophys., 24, 2619-2643, doi:10.5194/angeo-24-2619-2006, 2006.

Øieroset, M., Raeder, J., Phan, T. D., Wing, S., McFadden, J. P., Li, W., Fujimoto, M., Rème, H., and Balogh, A.: Global cooling and densification of the plasma sheet during an extended period of purely northward IMF on October 22 24, 2003, Geophys. Res. Lett., 32, L12S07, doi:10.1029/2004GL021523, 2005.

Øieroset, M., Phan, T. D., Angelopoulos, V., Eastwood, J. P., McFadden, J., Larson, D., Carlson, C. W., Glassmeier, K.-H., Fujimoto, M., and Raeder, J.: THEMIS multi-spacecraft observations of magnetosheath plasma penetration deep into the dayside low-latitude magnetosphere for northward and strong $B_{\mathrm{y}} \mathrm{IMF}$, Geophys. Res. Lett., 35, L17S11, doi:10.1029/2008GL033661, 2008.

Paularena, K. I., Richardson, J. D., Kolpak, M. A., Jackson, C. R., and Siscoe, G. L.: A dawn-dusk density asymmetry in Earth's magnetosheath, J. Geophys. Res., 106, 25377-25394, 2001.

Rème, H., Dandouras, I., Aoustin, C., Bosqued, J. M., Sauvaud, J. A., Vallat, C., Escoubet, P., Cao, J. B., Shi, J., BavassanoCattaneo, M. B., Parks, G. K., Carlson, C. W., Pu, Z., Klecker, B., Moebius, E., Kistler, L., Korth, A., Lundin, R., and the HIA team: The HIA instrument on board the Tan Ce 1 Double Star near-equatorial spacecraft and its first results, Ann. Geophys., 23, 2757-2774, doi:10.5194/angeo-23-2757-2005, 2005.

Song, P. and Russell, C. T.: Model of the Formation of the LowLatitude Boundary Layer for Strongly Northward Interplanetary Magnetic Field, J. Geophys. Res., 97, 1411-1420, 1992.

Spreiter, J. R. and Stahara, S. S.: Gasdynamic and magnetohydrodynamic modeling of the magnetosheath: A tutorial, Adv. Space Res., 14, 5-19, 1994.

Sundberg, T., Boardsen, S. A., Slavin, J. A., Blomberg, L. G., Cumnock, J. A., Solomon, S. C., Anderson, B. J., and Korth, H.: Reconstruction of propagating Kelvin-Helmholtz vortices at Mercury's magnetopause, Planet. Space Sci., 59, 2051-2057, 2011.

Sundberg, T., Boardsen, S. A., Slavin, J. A., Anaderson, B. J., Korth, H., Zurbuchen, T. H., Raines, J. M., Solomon, S. C.: MESSENGER orbital observation of large-amplitude KelvinHelmholtz waves at Mercury's magnetopause, J. Geophys. Res., 117, A04216, doi:10.1029/2011JA017268, 2012.

Takagi, K., Hashimoto, C., Hasegawa, H., Fujimoto, M., and TanDokoro, R.: Kelvin-Helmholtz instability in a magnetotail flanklike geometry: Three-dimensional MHD simulations, J. Geophys. Res., 11, A08202, doi:10.1029/2006JA011631, 2006.

Taylor, M. G. G. T. and Lavraud, B.: Observation of three distinct ion populations at the Kelvin-Helmholtz-unstable magnetopause, Ann. Geophys., 26, 1559-1566, doi:10.5194/angeo-261559-2008, 2008.

Taylor, M. G. G. T., Lavraud, B., C. P. Escoubet, Milan, S. E., Nykyri, K., Dunlop, M. W., Davies, J. A., Friedel, R. H. W., Frey, H., Bogdanova, Y. V., Åsnes, A., Laakso, H., Trávnicek, P., Masson, A., Opgenoorth, H., Vallat, C., Fazakerley, A. N., Lahiff, A. D., Owen, C. J., Pitout, F., Pu, Z., Shen, C., Zong, Q. G., Rème, H., Scudder, J., and Zhang, T. L.: The plasma sheet and boundary layers under northward IMF: A multi-point and multi-instrument perspective, Adv. Space Res., 41, 1619-1629, 
doi:10.1016/j.asr.2007.10.013, 2008.

Terasawa, T., Fujimoto, M., Mukai, T., Shinohara, I., Saito, Y., Yamamoto, T., Machida, S., Kokubun, S., Lazarus, A. J., Steinberg, J. T., and Lepping, R. P.: Solar Wind control of density and temperature in the near-Earth plasma sheet: WIND/Geotail collaboration, Geophys. Res. Lett., 24, 935-938, 1997.

Treumann, R. A.: Thoery of super-diffusion for the magnetopause, Geophys. Res. Lett., 24, 1727-1730, 1997.
Wei, C. Q. and Lee, L. C.: Coupling of Magnetopause-Boundary Layer to the Polar Ionosphere, J. Geophys. Res., 98, 5707-5725, 1993.

Wing, S. and Newell, P. T.: 2D plasma sheet ion density and temperature porfiles for northward and southward IMF, Geophys. Res. Lett., 29, 1307, doi:10.1029/2001GL013950, 2002. 